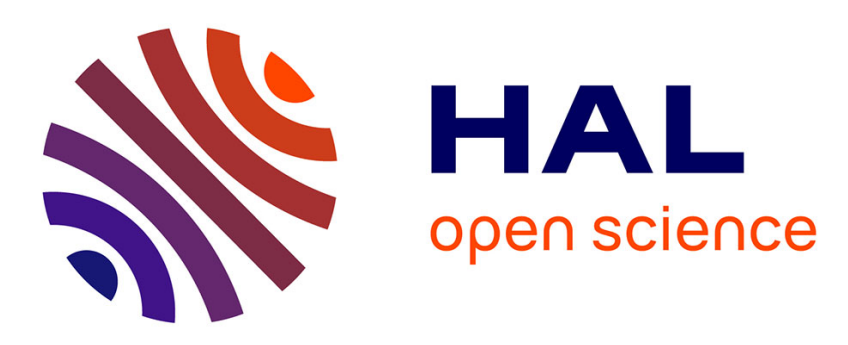

\title{
Theoretical investigation of the electronic relaxation in highly excited chrysene and tetracene: The effect of armchair vs zigzag edge
}

Evgeny Posenitskiy, Mathias Rapacioli, Didier Lemoine, Fernand Spiegelman

\section{- To cite this version:}

Evgeny Posenitskiy, Mathias Rapacioli, Didier Lemoine, Fernand Spiegelman. Theoretical investigation of the electronic relaxation in highly excited chrysene and tetracene: The effect of armchair vs zigzag edge. Journal of Chemical Physics, 2020, 152 (7), pp.074306. 10.1063/1.5135369 . hal02491991

\author{
HAL Id: hal-02491991 \\ https://hal.science/hal-02491991
}

Submitted on 26 Feb 2020

HAL is a multi-disciplinary open access archive for the deposit and dissemination of scientific research documents, whether they are published or not. The documents may come from teaching and research institutions in France or abroad, or from public or private research centers.
L'archive ouverte pluridisciplinaire HAL, est destinée au dépôt et à la diffusion de documents scientifiques de niveau recherche, publiés ou non, émanant des établissements d'enseignement et de recherche français ou étrangers, des laboratoires publics ou privés. 


\title{
Theoretical investigation of the electronic relaxation in highly-excited chrysene and tetracene: the effect of armchair versus zigzag edge
}

\author{
Evgeny Posenitskiy, ${ }^{1, \text { a) }}$ Mathias Rapacioli, ${ }^{2}$ Didier Lemoine, ${ }^{1}$ and Fernand Spiegelman ${ }^{2}$ \\ 1) Laboratoire Collisions Agrégats et Réactivité (LCAR), IRSAMC UMR5589, Université de Toulouse (UPS) and CNRS, \\ 118 Route de Narbonne, F-31062 Toulouse, France \\ ${ }^{2)}$ Laboratoire de Chimie et Physique Quantiques (LCPQ), IRSAMC UMR5626, Université de Toulouse (UPS) and CNRS, \\ 118 Route de Narbonne, F-31062 Toulouse, France
}

(Dated: 24 January 2020)

\begin{abstract}
Non-adiabatic molecular dynamics of neutral chrysene and tetracene molecules is investigated using Tully's fewest switches surface hopping algorithm coupled to the time-dependent density functional based tight binding method (TDDFTB) for electronic structure calculations. We first assess the performance of two DFTB parameters sets based on the computed TD-DFTB absorption spectra. Main focus is given to the analysis of the electronic relaxation from the brightest excited state following absorption of a UV photon. We determine the dynamical relaxation times and discuss the underlying mechanisms. Our results show that the electronic population of the brightest excited singlet state in armchair-edge chrysene decays an order-of-magnitude faster than the one in zigzag-edge tetracene. This is correlated with a qualitatively similar difference of energy gaps between the brightest state and the state lying just below in energy, which is also consistent with our previous study on polyacenes.
\end{abstract}

\section{INTRODUCTION}

Polycyclic Aromatic Hydrocarbon molecules (PAHs) are known to play a role in various domain of chemistry and physics such as combustion ${ }^{1-5}$, atmospheric and environmental chemistry ${ }^{6-9}$ and interstellar physical chemistry ${ }^{10-18}$ PAHs are supposed to contribute to a large amount of the carbonaceous matter in the interstellar medium and have been proposed as the carriers of unidentified IR bands ${ }^{12,13}$ and the precursors of the Diffuse Interstellar Bands ${ }^{19-21}$. The specific assignment of these bands to specific molecules or compounds is still not clear, mostly due to the fact that despite they all present an aromatic character, their organization is multi-fold in term of size (from a pair of cycles to a few hundreds) and moreover, for a given size, they can organize in various architectures such as cata-condensed (1D) or peri-condensed (2D) structures based on the hexagonal motif. Their characterization obviously relies on spectroscopic properties, either in the infra-red or in the electronic domain (absorption/emission).

The ground state properties of PAH molecules have been the object of numerous studies both with experimental and theoretical approaches, with focus on structural, thermodynamical and vibrational properties. However both in atmospheric conditions or in the interstellar medium, $\mathrm{PAH}$ molecules are exposed to radiation and excited electronic states certainly play an important role in influencing their dynamical properties, manifesting via various processes such as photo-formation, relaxation, photo-fragmentation, photoreactivity. A number of studies were concerned with the determination of their visible/UV electronic spectrum. ${ }^{22-30}$ Theoretical approaches to compute excited states are either wavefunction-type methods based on variational configuration interaction possibly completed by perturbative schemes ${ }^{31}$ or Time-Dependent Density Functional Theory (TD-DFT)

a)Electronic mail: posenitskiy @irsamc.ups-tlse.fr schemes $^{32-34}$. Beyond the determination of vertical spectra, experimental setups have been designed, to explore the postexcitation dynamics of systems as large as PAHs with femtosecond resolution. ${ }^{35-37}$ From the theoretical side, a number of studies have been focused on the static calculations of excited states in cationic PAHs and their possible non-radiative relaxation towards the ground state. ${ }^{38-40}$ More recently, multi-state non-adiabatic molecular dynamics (NAMD) of small cationic polyacenes have been investigated, coupling the electronic and nuclei motion. ${ }^{41-44}$ The coupling of TD-DFT dynamics with non-adiabatic nuclei dynamics is a promising approach to investigate dynamics in excited states. ${ }^{45}$ However it is still too computationally demanding in order to be able to handle reasonably large PAH systems with the goal to reach statistical convergence of the dynamical properties and evolution. A further complexity is provided by the fact that the larger the molecule, the higher the density of electronic states and, accordingly, the larger number of states to be included in the NAMD simulation (at least all those lying below the absorbing one considered). An alternative approach is offered by the Time-Dependent Density Functional Based Tight-Binding (TD-DFTB) method, pioneered by Niehaus et al. ${ }^{46}$ and later combined with the Tully's fewest-switches trajectory surface hopping (FSSH) scheme ${ }^{47,48}$ by several groups. ${ }^{49-52}$

Recently, we reported a study of non-adiabatic electronnuclei relaxation processes in polyacenes using an implementation of FSSH/TD-DFTB molecular dynamics developed within the framework of the deMon-Nano package. ${ }^{53}$ The work was essentially concerned with the investigation of size dependence of the relaxation in polyacenes. In our former work, only the zigzag-edge cata-condensed morphology characterizing polyacenes was investigated. ${ }^{52}$ Yet, the armchairedge cata-condensed morphology characterizing phenacenes is also of interest and chrysene is the first in the series with four aromatic cycles. Furthermore in the case of the tetracene and chrysene isomers, it is worth noticing that their respective experimental absorption spectra are quite similar, both showing a strong absorption around $270 \mathrm{~nm} .{ }^{24,54-56}$ Given this 
similarity, the investigation and comparison of their respective relaxation dynamics should prove invaluable in unraveling shape- and/or symmetry-dependent effects. The present paper is devoted to a FSSH/TD-DFTB investigation of the post-excitation dynamics following excitation of tetracene and chrysene in their excited electronic state with the largest absorbing intensity from the ground state ("brightest state"). Section II is devoted to a brief reminder of the NAMD method and some computational details. The static and dynamical results are reported and discussed in Section III. Finally conclusions and implications are outlined.

\section{METHODS AND COMPUTATIONAL DETAILS}

In this section, we present a brief outline of the TD-DFTB formalism and the Tully's fewest-switches trajectory surface hopping scheme, which is implemented in the open-source deMon-Nano ${ }^{53}$ code.

The self-consistent charge DFTB (SCC-DFTB) was developed by Elstner et al. ${ }^{57}$ as an extension of the original DFTB framework. ${ }^{58,59}$ It is based on the 2-nd order expansion of the Kohn-Sham DFT total energy functional around a reference electronic density $n_{0}$, so the final expression for the DFTB total energy reads

$$
E_{\mathrm{SCC}}=\sum_{i=1}^{N_{\mathrm{occ}}} n_{i} \sum_{\mu \nu} a_{\mu i} H_{\mu \nu}^{0} a_{v i}+\frac{1}{2} \sum_{A=1}^{N_{\mathrm{at}}} \sum_{B=1}^{N_{\mathrm{at}}} \Delta q_{A} \gamma_{A B} \Delta q_{B}+E_{\mathrm{rep}},
$$

where $n_{i}$ is the occupation number of molecular orbital (MO) $i, \mu$ and $v$ are the Kohn-Sham atomic orbital indices, $\Delta q_{A}$ is the Mulliken charge of atom A, $E_{\text {rep }}$ is the atomic pair repulsive contribution, $a_{\mu i}$ are Kohn-Sham MO coefficients and $\gamma_{A B}$ describes the Coulomb interaction between spherically symmetric charge distributions centered on the atoms A and $\mathrm{B}$ with a short range exchange-correlation contribution. The total energy $E_{\mathrm{SCC}}$ is further minimized following the selfconsistent procedure as proposed by Elstner et al..$^{57}$

Linear response TD-DFTB was developed by Niehaus et $a l .{ }^{46}$ as a DFTB analogue of the conventional linear response TD-DFT. ${ }^{33}$ Excitation energies are given as eigenvalues $\Omega_{I}$ of the following matrix equation:

$$
\left(\begin{array}{ll}
\mathbf{A} & \mathbf{B} \\
\mathbf{B} & \mathbf{A}
\end{array}\right)\left(\begin{array}{l}
\mathbf{X} \\
\mathbf{Y}
\end{array}\right)=\Omega_{I}\left(\begin{array}{cc}
\mathbb{1} & 0 \\
0 & -\mathbb{1}
\end{array}\right)\left(\begin{array}{l}
\mathbf{X} \\
\mathbf{Y}
\end{array}\right),
$$

where $\mathbb{1}$ is the identity matrix, $\mathbf{A}$ and $\mathbf{B}$ are matrices with the elements given by

$$
\begin{aligned}
A_{i a, j b}= & \left(\varepsilon_{a}-\varepsilon_{i}\right) \delta_{i j} \delta_{a b}+2 K_{i a, j b} ; \\
& B_{i a, j b}=2 K_{i a, j b} ;
\end{aligned}
$$

and indices $i, j$ and $a, b$ denoting the occupied and virtual MOs with energies $\varepsilon_{i}$ and $\varepsilon_{a}$, respectively. The coupling matrix elements $K_{i a, j b}$ are calculated within the DFTB approach using the generalized Mulliken approximation. ${ }^{46}$

Standard molecular dynamics usually relies on the BornOppenheimer approximation, which retains a single adiabatic electronic state in the expansion of the wavefunction of the system. However, nuclear and electronic degrees of freedom evolve with similar timescales in the regions where two or more electronic potential energy surfaces (PES) are getting close (e.g. in the vicinity of conical intersection). This is a well-known breakdown of the Born-Oppenheimer (adiabatic) approximation. Several approaches have been developed in recent years to include the non-adiabatic effects in the dynamics both at the $a b$-initio ${ }^{60}$ and mixed quantum-classical ${ }^{61}$ levels of theory. In this article, we use Tully's FSSH scheme. ${ }^{48}$ It is a quantum-classical approach to quantum dynamics, with the nuclear wavepacket motion simulated by an ensemble of independent classical trajectories, each one evolving on a single electronic state at a given time.

The determinations of the switching probabilities between adiabatic states is controlled by the electron dynamics. Substituting the electronic wavefunction expanded in a basis of adiabatic electronic states into the time-dependent electronic Schrödinger equation, one derives the following equation for the propagation of the complex expansion coefficients $C_{J}(t)$ :

$$
i \hbar \frac{d C_{J}(t)}{d t}=C_{J}(t) E_{J}(t)-i \hbar \sum_{K \neq J} C_{K}(t) D_{J K}(t),
$$

where $E_{J}$ is the adiabatic energy of state $J, D_{J K}$ is the nonadiabatic coupling between states $J$ and $K$, which is calculated using a finite difference method. ${ }^{48}$ In our implementation, the non-adiabatic coupling is calculated using a finite difference method: 48

$$
D_{J K}(t+\Delta t / 2) \approx \frac{\left\langle\psi_{J}(t) \mid \psi_{K}(t+\Delta t)\right\rangle-\left\langle\psi_{J}(t+\Delta t) \mid \psi_{K}(t)\right\rangle}{2 \Delta t} .
$$

In the equation above, wavefunction overlaps $\left\langle\psi_{J}(t) \mid \psi_{K}(t+\Delta t)\right\rangle$ can be computed based on TD-DFTB eigenvectors and singly excited Slater determinants following Ref. 49.

It is important to apply the decoherence correction on $C_{J}$ since the propagation of Eq. (5) in FSSH is overcoherent, which means that electronic coherences $C_{I} C_{J}^{*}$ do not vanish after passing through the region of strong coupling between states $I$ and $J$. Decoherence corrections have been shown to be crucial in a number of applications. ${ }^{62,63}$ We use the simplified decay of mixing method to correct the $C_{J}$ coefficients. ${ }^{62,64}$

The probability to switch from the active state $I$ to another state $K$ during the electronic time step $\Delta \tau$ is estimated from the following equation: ${ }^{50}$

$$
P_{I \rightarrow K}(\tau)=\max \left[0 ;-2 \Delta \tau \frac{\operatorname{Re}\left(C_{I}^{*}(\tau) C_{K}(\tau)\right)}{\left|C_{I}\right|^{2}} D_{I K}(\tau)\right],
$$

where $\left|C_{I}\right|^{2}$ is the electronic population of a given excited state $I$. More details regarding an implementation of the Tully's FSSH scheme coupled to the TD-DFTB approach can be found in Refs. 49-52, which follows the original idea of Mitrić et al. from Ref. 65.

To conserve the total energy after hopping, the nuclear velocities are rescaled uniformly by a factor $\beta$ following the energy conservation law:

$$
E_{I}+E_{\mathrm{kin}}=E_{J}+\beta^{2} E_{\mathrm{kin}} .
$$


The first part of the discussion in the next section is devoted to the comparison of TD-DFTB and TD-DFT absorption spectra. The TD-DFT absorption spectra were computed with Gaussian 09 package ${ }^{66}$ using BLYP functional and 6$31 \mathrm{G}(\mathrm{d}, \mathrm{p})$ basis set while the TD-DFTB data were calculated with the deMon-Nano ${ }^{53}$ code using both matsci-0-3 ${ }^{67}$ and mio-1-1 ${ }^{57}$ sets of parameters.

Eq. (5) is integrated using a 4-th order Runge-Kutta algorithm with an electronic time step $\Delta \tau=0.048$ as. Each classical trajectory is propagated using a time step $\Delta t=0.25$ fs during 300 fs using matsci-0-3 ${ }^{67}$ DFTB parameters set for the electronic structure calculations. The choice of the latter basis set is justified in the next section. The most straightforward way to avoid singularities of non-adiabatic coupling at the point of conical intersection is to swap states once the energy gap between them drops below a given threshold. In our simulations, we use a threshold of $1 \mathrm{meV}$ for conical intersection between excited states and $0.1 \mathrm{eV}$ to detect the crossing between the lowest excited and ground states. The latter value was taken larger due to the inappropriate topology of $S_{1} / S_{0}$ PES in TD-DFT. ${ }^{68}$

Initial conditions were sampled from the thermal distribution of the ground state. Single trajectory was equilibrated at $T=300 \mathrm{~K}$ during $50 \mathrm{ps}$ using a chain of 5 Nosé-Hoover thermostats and $0.5 \mathrm{fs}$ time step. Snapshots were taken every 50 fs to be further used as initial conditions for the excited states dynamics. We applied an additional constraint by setting the overall angular momentum of the system to zero at each step. This is crucial for FSSH since the velocity rescaling scheme used in this work does not conserve the total angular momentum (unless it is equal to zero). This is not an issue if an energy gradient between two states that contribute to the hopping event is used to rescale the nuclear velocities. ${ }^{69}$

In this work, we apply a fitting procedure different from the one used in our previous work. Instead of using a simple exponent (e.g. $\exp (-t / \tau)$ ) to extract the decay time $\tau$, we implement the following equation to fit the population of the initially excited state (IS):

$$
f(t)=A+B \exp (-t / \tau),
$$

where $A=\min \left[P_{\mathrm{IS}}(t)\right] /\left(1+\min \left[P_{\mathrm{IS}}(t)\right]\right)$ and $B=1 /(1+$ $\left.\min \left[P_{\mathrm{IS}}(t)\right]\right)$. This new fitting procedure, adapted from the work of Marciniak et $a l .{ }^{37}$, allows one to fit the initial population more accurately thanks to the shift $A$, which takes into account the non-vanishing population at the end of the simulation (e.g. non-Kasha behaviour).

\section{RESULTS AND DISCUSSION}

\section{A. Absorption spectra of chrysene and tetracene}

A qualitative assignment of the absorption spectra of chrysene and tetracene has been presented quite a long time ago. $^{70,71} \mathrm{We}$ first assess the accuracy of TD-DFTB absorption spectra computed for chrysene and tetracene with matsci-0-3 (MAT) and mio-1-1 (MIO) sets of parameters. Both systems are considered at their respective DFTB equilibrium geometries, namely with $D_{2 h}$ symmetry for tetracene and $C_{2 h}$ for chrysene.

As far as we know, the TD-DFTB absorption spectrum of chrysene has not been published before. This is why we decided to study it in more details and to compare two commonly used DFTB sets of parameters. As for tetracene, the TD-DFTB spectrum has already been published with both $\mathrm{MIO}^{46}$ and $\mathrm{MAT}^{52}$ parameters. Therefore, it is not been shown here, even though we have repeated these calculations in order to check that the MAT set is better suited for our study.

In the case of chrysene, the results obtained with MIO and MAT parameters are relatively similar (see Figure 1). However, the bands computed with MAT are blue-shifted as compared to the TD-DFT ones. Thus, the MIO set predicts better the positions of the TD-DFT absorbing bands, but the brightest band computed at $4.61 \mathrm{eV}(269 \mathrm{~nm})$ with the MAT set is closer to the experimental one at $270 \mathrm{~nm} .{ }^{54}$ Both parameters sets show that the brightest excited state $\left(S_{8}\right)$ is dominated (with a weight of 0.59 and 0.62 for MIO and MAT sets, respectively) by the HOMO- $\rightarrow$ LUMO +1 transition (see Figures $3 \mathrm{a}$ and $3 \mathrm{c}$ ) with an oscillator strengths equal to 0.49 and 0.56 for MIO and MAT sets, respectively. We have performed additional TD-DFT calculations for chrysene with B3LYP and CAM-B3LYP (see Tables S4 and S5, respectively, in the Supplementary Material) functionals. Indeed, TD-DFTB shows a poor agreement with these functionals. In particular, both B3LYP and CAM-B3LYP spectra are strongly blue-shifted compared to TD-DFTB and TD-DFT with BLYP functional. Notably, CAM-B3LYP fails to detect two bright states with relatively close oscillator strengths that are present in TDDFTB, TD-DFT/BLYP and experimental ${ }^{54-56}$ data. It shows a single very bright state with 1.66 oscillator strength at 5.24 eV.

There is a significant difference in the absorption spectra of tetracene. The brightest excited states computed with MAT and MIO basis sets are $S_{7}$ and $S_{10}$, respectively, even though the dominant (with a weight of 0.55 ) transition is $\mathrm{HOMO} \rightarrow \mathrm{LUMO}+2$ (see Figures $3 \mathrm{~b}$ and $3 \mathrm{~d}$ ) in both cases. This is clearly an indication that the splitting of degenerate states induced by TD-DFTB coupling matrix in tetracene is stronger for the MIO set. This results in a shift of some higherlying excited states below the brightest one. TD-DFT predicts the brightest absorbing band to be $S_{7}$ with the same dominant $\mathrm{HOMO} \rightarrow \mathrm{LUMO}+2$ transition as in TD-DFTB. Thus, we conclude that the MAT basis is more reliable for the simulation of the non-adiabatic dynamics in tetracene, which is likely to be affected by a wrong topology of excited PES. This set of parameters has been used in this work to study relaxation mechanisms in both chrysene and tetracene.

\section{B. Electronic relaxation from the brightest excited state}

All trajectories considered below were launched in the brightest, namely that with the largest oscillator strength, excited singlet state of the corresponding molecule ( $S_{8}$ for chrysene and $S_{7}$ for tetracene), at their respective ground state 
equilibrium geometries. It is worth mentioning that the brightest absorbing band is located close to $4.6 \mathrm{eV}$ in both chrysene and tetracene (see Figure 2). Thus, we will present the results of FSSH launched in these particular states to compare the relaxation mechanisms as well as computed decay times.

FSSH results obtained with 63 trajectories launched in the brightest excited state of tetracene have been reported in Ref. 52. We have repeated these calculations with 100 trajectories to assess the convergence as well as the accuracy of our results. Population curves averaged over 100 trajectories (see Figure 4) are qualitatively similar to the ones reported in Ref. 52 for 63 trajectories and the computed decay time of $64 \mathrm{fs}$ is in a very good agreement with 65 fs calculated using a new fitting procedure for the populations presented in our previous work.

FSSH of chrysene has been simulated in this work for the first time. Relaxation from the brightest excited state of chrysene (see Figure 5) differs drastically from the one in tetracene. First of all, the computed decay time is $7 \mathrm{fs}$, which is an order-of-magnitude smaller than in tetracene. This is consistent with the rapid transfer of the initial population to the bunch of low-lying excited states, which ends up about $2 \%$ at the end of propagation compared to $8 \%$ in tetracene. In order to demonstrate that an observed ultrafast relaxation in chrysene is not a direct consequence of the selected energy gap threshold, we have performed additional calculations under the same conditions, yet changing the threshold to 0.1 or $10 \mathrm{meV}$ (see Figure S2 in the Supplementary Material). The population curves are not drastically affected by these changes and the computed decay time of the $S_{8}$ state in both cases is approximately $7 \mathrm{fs}$, which is in a very good agreement with the value computed for $1 \mathrm{meV}$ threshold. We have also performed calculations for chrysene with the MIO set of DFTB parameters, using an ensemble of 89 trajectories launched in the brightest excited $S_{8}$ state and propagated during $300 \mathrm{fs}$ (see Figure S1 in the Supplementary Material). The computed decay time is approximately $8 \mathrm{fs}$, which is in a good agreement with 7 fs calculated with the MAT basis.

We would like to emphasize that the fractional occupation (percentage of trajectories residing in a given state at a given time) of the state below the brightest one is $7 \%$ in tetracene and $3 \%$ in chrysene after $300 \mathrm{fs}$ of propagation. This can be due to a partial trapping of the population in some higherlying excited states of tetracene, which is also consistent with the delayed decay of the initial state.

It is worth mentioning that an ensemble of 100 trajectories allows us to resolve only processes that occur with a probability greater than $10 \%{ }^{61}$ However, we have noticed in our previous study ${ }^{52}$ that going from 63 to 127 trajectories does not change significantly the population curves and the computed decay times of naphthalene and anthracene. This is also true for tetracene when going from 63 to 100 trajectories as indicated in Table I. Indeed, 100 trajectories is not enough to draw solid conclusions about some processes like photoisomerisation or intersystem crossings. Nevertheless, we believe that our results can be used to qualitatively describe the rapid internal conversion between excited singlet states and to understand the decay mechanisms of the brightest states.

\section{Discussion}

The results of our calculations are summarized in Table I.

In order to explain the difference in decay times and underlying relaxation mechanisms, we computed the energy gaps along each trajectory between the initial state and the neighbouring one with lower energy for both tetracene and chrysene (see Figure 6). It is clear from Figure 6 that at the start of dynamics all trajectories in chrysene either go to the region of small gaps $(<0.1 \mathrm{eV})$ or through the conical intersection. Thus, there is a funnel, which drives the rapid population transfer from the initial state. On the contrary, in tetracene, very few trajectories reach the region of small gaps and the average gap remains twice larger than the one in chrysene. A similar analysis was used to explain changes in decay times with respect to the size of polyacenes. ${ }^{52}$ It also correlates well with the energy gap computed at the equilibrium geometry (see Table I). This initial gap turns out to be roughly conserved during the short time phase of the trajectory and can be used as a first order approximation for estimating the decay times. Consistently, the number of surface hops is larger in chrysene than in tetracene. In addition to the notably-differing relative gaps between excited states, the symmetry of the initial chrysene geometry $\left(C_{2 h}\right)$ is lower than that for tetracene $\left(D_{2 h}\right)$, and therefore yields more non-zero non-adiabatic coupling elements, which may also enhance the electronic relaxation and thus contribute to the much faster decay.

There is clearly a violation of the Kasha's rule ${ }^{72}$ for the rapid decay to the $S_{1}$ state in both tetracene and chrysene within the considered timescale. Indeed, $300 \mathrm{fs}$ is a relatively short timescale for a complete relaxation. Nevertheless, a partial trapping of the population has been observed for some excited states that might lead to an incomplete relaxation towards the lowest excited state. Deviations from Kasha's rule have been reported in several studies. ${ }^{50,73}$ A detailed discussion of this issue is given elsewhere. ${ }^{50,52}$

The results of this paper can also be used for a qualitative analysis of the emission of photoexcited chrysene and tetracene. In particular, we have noticed that the major fraction (53\%) of trajectories ends up in excited state $S_{5}$ with $24 \%$ shared equally between states $S_{2}$ and $S_{3}$ after 300 fs of propagation. This partial trapping in $S_{5}$ state is pronounced and can lead to significant delay in the non-adiabatic relaxation. However, in chrysene the situation is changing drastically and we observe $36 \%$ of trajectories in $S_{1}, 24 \%$ shared equally between $S_{2}$ and $S_{3}$ and $27 \%$ in $S_{4}$ at the end of simulation. Occupation of all excited singlet states at the end of the simulation is summarized in Figure 7.

Last, we would like to understand which role does the nuclear motion play in the relaxation of excited chrysene and tetracene. To address this question, we compiled in Figure 8 the geometrical configurations of each isomer, extracted at the end of each of the $100 \mathrm{FSSH}$-trajectory simulations. It is clear that both chrysene and tetracene experience strong out-of-plane motion, which is the major deactivation channel during the relaxation. This motion breaks the corresponding symmetry of the molecule, thereby increasing the coupling between excited states. Nuclear displacements along 
TABLE I. Summary of the FSSH simulations for chrysene and tetracene. Energies and positions of excited states are taken from the TD-DFTB spectra computed with MAT set of parameters at the ground state equilibrium geometry. IS (B) stands for the initial (brightest) excited state.

\begin{tabular}{llcccc}
\hline \hline Molecule & IS & $\Omega_{\text {IS }}(\mathrm{eV})$ & Number of trajectories & Decay time of IS (fs) & Energy gap B//B-1 (eV) \\
\hline Chrysene & $S_{8}$ & 4.61 & 100 & 7 & 0.05 \\
\hline Tetracene & $S_{7}$ & 4.56 & 100 & 64 & 0.22 \\
\hline Tetracene $^{\mathrm{a}}$ & $S_{7}$ & 4.56 & 63 & 65 & 0.22 \\
\hline \hline
\end{tabular}

a Adapted from Ref. 52

the $x, y$ and $z$ axes have been computed for each geometry with respect to the DFTB ground state equilibrium configuration. The mean (maximum) displacements along $x$ and $y$ (in the molecular plane) are respectively $0.04(0.31) \AA$ and 0.05 (0.31) $\AA$ in chrysene and 0.04 (0.33) $\AA$ and $0.04(0.30) \AA$ in tetracene. Notably, the mean (maximum) displacements along the perpendicular axis $z$ are 0.14 (1.07) $\AA$ and $0.14(0.99) \AA$ in chrysene and tetracene, respectively. These values are significantly larger than the ones computed along $x$ and $y$, which is consistent with the strong out-of-plane perturbations visible in Figure 8. Considering polyacenes and phenacenes as rather rigid molecules (at least concerning their carbonaceous skeleton), we expect the out-of-plane motion to remain the leading deactivation channel during the relaxation of larger cata-condensed PAHs.

\section{CONCLUSION}

In this article, a detailed theoretical study of the nonadiabatic molecular dynamics launched in the brightest singlet excited state has been presented for neutral chrysene and tetracene molecules. The results have been obtained using Tully's FSSH scheme coupled to the TD-DFTB formalism for electronic structure calculations.

We have first assessed the performance of two DFTB parameters sets by calculating the absorption spectra of chrysene and tetracene and upon comparison with TD-DFT and available experimental data. Our conclusion is that the MAT set of DFTB parameters ${ }^{67}$ is more reliable, especially when the brightest excited singlet state has to be computed.

Relaxation from the brightest singlet excited states via the cascade of radiationless transitions is reported for armchairshaped chrysene and zigzag-shaped tetracene. The detailed analysis reveals an order-of-magnitude difference in decay times of the initial electronic populations (brightest excited states) between the two molecules. This difference is correlated with the energy gap between the initially excited state and the neighbouring one with lower energy, which is consistent with our previous study on neutral polyacenes. ${ }^{52}$ The much faster decay rate for armchair-edge chrysene may also be enhanced by the lesser symmetry induced with respect to zigzag-edge tetracene. One should nevertheless precise the limits of the present conclusions. TD-DFTB neither involves doubly excited states, nor Rydberg states. Also, interband transitions via spin-orbit coupling have not been considered here. They are not supposed to be dominant since spin-orbit coupling is expected to be small for light elements, never- theless they have been mentioned to be active in some specific cases. ${ }^{74-76}$ We however expect the main conclusions to hold for short relaxation times. This shape dependence of the relaxation process should obviously also influence the long term populations of chrysene versus tetracene in the interstellar medium.

This research contributes to the understanding of the photophysics of PAHs and, more particularly, sheds light on the effect, which different shapes may introduce in the excited states dynamics. It also provides promising insights on the photostability of the considered molecules following the absorption of a UV photon, which is of interest for astronomers (e.g. for understanding the evolution of PAHs in the photodissociation regions), atmospheric chemistry and laboratory experiments concerned with atto- or femto-second laser spectroscopy of these compounds. Referring more particularly to species in the interstellar medium, electronic absorption band widths can be related to the relaxation timescales ${ }^{42}$ and both the shape-dependent relaxation mechanisms predicted in the present work and the different final state populations at the end of the dynamical processes might contribute to the assignment of emission spectra. Finally, it would certainly be interesting to investigate whether such differentiation is an isolated case property or if it extends to larger cata-condensed phenacenes versus polyacenes.

\section{SUPPLEMENTARY MATERIAL}

See supplementary material for the electronic populations of excited singlet states of neutral chrysene computed with MIO set of DFTB parameters (Figure S1) and with 0.1 or 10 meV energy gap thresholds (Figure S2); TD-DFTB absorption spectra of neutral chrysene computed with MIO (Table S1) and MAT (Table S2) sets of DFTB parameters; TD-DFT absorption spectra of neutral chrysene computed with BLYP (Table S3), B3LYP (Table S4) and CAM-B3LYP (Table S5) functionals.

\section{ACKNOWLEDGMENTS}

The authors would like to thank Bruno Lepetit for many fruitful discussions. We acknowledge the European Union (EU) and Horizon 2020 funding awarded under the Marie Skłodowska-Curie action to the EUROPAH consortium, grant number 722346 . This work was performed using HPC resources from CALMIP (Grant 2019-P18019). We are grate- 
ful to the referees for their insightful comments on the manuscript.

${ }^{1}$ J. P. Longwell, "The formation of polycyclic aromatic hydrocarbons by combustion," Symposium (International) on Combustion 19, 1339-1350 (1982).

${ }^{2}$ P. Liu, Z. Li, and W. L. Roberts, "The growth of pahs and soot in the post-flame region," Proceedings of the Combustion Institute 37, 977-984 (2019).

${ }^{3}$ Q. Mao, A. C. T. van Duin, and K. H. Luo, "Formation of incipient soot particles from polycyclic aromatic hydrocarbons: A reaxff molecular dynamics study," Carbon 121, 380-388 (2017).

${ }^{4} \mathrm{~B}$. Saha, S. Irle, and K. Morokuma, "Formation mechanism of polycyclic aromatic hydrocarbons in benzene combustion: Quantum chemical molecular dynamics simulations," The Journal of Chemical Physics 132, 224303 (2010).

${ }^{5}$ B. V. Unterreiner, M. Sierka, and R. Ahlrichs, "Reaction pathways for growth of polycyclic aromatic hydrocarbons under combustion conditions, a dft study," Physical Chemistry Chemical Physics 6, 4377-4384 (2004).

${ }^{6} \mathrm{~K}$. Ravindra, R. Sokhi, and R. Van Grieken, "Atmospheric polycyclic aromatic hydrocarbons: Source attribution, emission factors and regulation," Atmospheric Environment 42, 2895-2921 (2008).

${ }^{7}$ Y. Zhang and S. Tao, "Global atmospheric emission inventory of polycyclic aromatic hydrocarbons (pahs) for 2004," Atmospheric Environment 43, 812-819 (2009).

${ }^{8}$ Y.-z. An, X. Li, S.-p. Teng, K. Wang, Y.-q. Pei, J. Qin, and H. Zhao, “Development of a soot particle model with pahs as precursors through simulations and experiments," Fuel 179, 246-257 (2016).

M. S. Callén, M. T. de la Cruz, J. M. López, R. Murillo, M. V. Navarro, and A. M. Mastral, "Some inferences on the mechanism of atmospheric gas/particle partitioning of polycyclic aromatic hydrocarbons (pah) at zaragoza (spain)," Chemosphere 73, 1357-1365 (2008).

${ }^{10}$ A. G. G. M. Tielens, "Interstellar polycyclic aromatic hydrocarbon molecules," Annual Review of Astronomy and Astrophysics 46, 289-337 (2008).

${ }^{11}$ G. P. van der Zwet and L. J. Allamandola, "Polycyclic aromatic hydrocarbons and the diffuse interstellar bands," Astron. Astrophys. 146, 76-80 (1985).

${ }^{12}$ L. J. Allamandola, A. G. G. M. Tielens, and J. R. Barker, "Polycyclic aromatic-hydrocarbons and the unidentified infrared-emission bands - auto exhaust along the milky-way," Astrophys. J. 290, L25-L28 (1985).

${ }^{13}$ A. Léger and J. L. Puget, "Identification of the 'unidentified' IR emission features of interstellar dust?" Astron. Astrophys. 137, L5-L8 (1984).

${ }^{14}$ C. Joblin, L. D'Hendecourt, A. Leger, and D. Defourneau, "Infrared spectroscopy of gas-phase pah molecules i. role of the physical environment," Astronomy and Astrophysics 281, 923-936 (1993).

${ }^{15} \mathrm{~J}$. Oomens, A. G. G. M. Tielens, B. G. Sartakov, G. von Helden, and G. Meijer, "Laboratory infrared spectroscopy of cationic polycyclic aromatic hydrocarbon molecules," The Astrophysical Journal 591, 968-985 (2003).

${ }^{16}$ S. R. Langhoff, "Theoretical infrared spectra for polycyclic aromatic hydrocarbon neutrals, cations, and anions," The Journal of Physical Chemistry 100, 2819-2841 (1996).

${ }^{17}$ C. W. Bauschlicher, E. Peeters, and L. J. Allamandola, "Infrared spectra of very large irregular polycyclic aromatic hydrocarbons (pahs)," The Astrophysical Journal 697, 311-327 (2009).

${ }^{18}$ M. Alliati, D. Donaghy, X. Tu, and J. W. Bradley, "Ionic species in a naphthalene plasma: Understanding fragmentation patterns and growth of pahs,' The Journal of Physical Chemistry A 123, 2107-2113 (2019).

${ }^{19}$ A. Léger and L. D'Hendecourt, "Are polycyclic aromatic hydrocarbons the carriers of the diffuse interstellar bands in the visible?" Astron. Astrophys. 146, 81-85 (1985).

${ }^{20}$ F. Salama, E. L. O. Bakes, L. J. Allamandola, and A. G. G. M. Tielens, "Assessment of the polycyclic aromatic hydrocarbon-diffuse interstellar band proposal," Astrophys. J. 458, 621 (1996).

${ }^{21}$ F. Salama, G. A. Galazutdinov, J. Krełowski, L. J. Allamandola, and F. A. Musaev, "Polycyclic aromatic hydrocarbons and the diffuse interstellar bands: A survey,” Astrophys. J. 526, 265-273 (1999).
${ }^{22}$ H. H. Heinze, A. Görling, and N. Rösch, "An efficient method for calculating molecular excitation energies by time-dependent density-functional theory," The Journal of Chemical Physics 113, 2088-2099 (2000).

${ }^{23}$ F. Bettanin, L. F. A. Ferrão, M. Pinheiro, A. J. A. Aquino, H. Lischka, F. B. C. Machado, and D. Nachtigallova, "Singlet la and $\mathrm{lb}$ bands for $\mathrm{n}$ acenes $(n=2-7)$ : A casscf/caspt2 study," Journal of Chemical Theory and Computation 13, 4297-4306 (2017).

${ }^{24} \mathrm{H}$.-H. Perkampus, $U V$-vis atlas of organic compounds, 2 nd ed. (Weinheim: $\mathrm{VCH}, 1992)$ p. $1525 \mathrm{pp}$.

${ }^{25}$ S. Hirata, T. Lee, and M. Head-Gordon, "Time-dependent density functional study on the electronic excitation energies of polycyclic aromatic hydrocarbon radical cations of naphthalene, anthracene, pyrene, and perylene,” J. Chem. Phys. 111, 8904-8912 (1999).

${ }^{26}$ S. Hirata, M. Head-Gordon, J. Szczepanski, and M. Vala, "Time-dependent density functional study of the electronic excited states of polycyclic aromatic hydrocarbon radical ions.” J. Phys. Chem. A 107, 4940-4951 (2003).

${ }^{27}$ G. Malloci, G. Mulas, G. Cappellini, and C. Joblin, "Time-dependent density functional study of the electronic spectra of oligoacenes in the charge states $-1,0,+1$, and +2 ," Chem. Phys. 340, 43-58 (2007).

${ }^{28}$ G. Malloci, G. Mulas, C. Cecchi-Pestellini, and C. Joblin, "Dehydrogenated polycyclic aromatic hydrocarbons and uv bump," Astron. Astrophys. 489, 1183-1187 (2008).

${ }^{29}$ J. Weisman, T. Lee, F. Salama, and M. Head-Gordon, "Time-dependent density functional theory calculations of large compact polycyclic aromatic hydrocarbon cations: Implications for the diffuse interstellar bands," Astrophys. J. 587, 256-261 (2003).

${ }^{30}$ M. Rapacioli, A. Simon, C. C. Marshall, J. Cuny, D. Kokkin, F. Spiegelman, and C. Joblin, "Cationic methylene-pyrene isomers and isomerization pathways : Finite temperature theoretical studies," J. Phys. Chem. A 119 9089-9100 (2015).

${ }^{31}$ T. Helgaker, P. Jørgensen, and J. Olsen, Molecular Electronic-Structure Theory (John Wiley \& Sons, Ltd, 2000) p. 938pp.

${ }^{32}$ R. E. Stratmann, G. E. Scuseria, and M. J. Frisch, "An efficient implementation of time-dependent density-functional theory for the calculation of excitation energies of large molecules," The Journal of Chemical Physics 109, 8218-8224 (1998).

${ }^{33}$ M. E. Casida, "Time-dependent density-functional theory for molecules and molecular solids," Journal of Molecular Structure: THEOCHEM 914, 3-18 (2009).

${ }^{34}$ E. Runge and E. K. U. Gross, "Density-functional theory for timedependent systems," Physical Review Letters 52, 997-1000 (1984).

${ }^{35}$ A. Marciniak, V. Despré, T. Barillot, A. Rouzée, M. C. E. Galbraith, J. Klei, C. H. Yang, C. T. L. Smeenk, V. Loriot, S. N. Reddy, A. G. G. M. Tielens, S. Mahapatra, A. I. Kuleff, M. J. J. Vrakking, and F. Lépine, "Xuv excitation followed by ultrafast non-adiabatic relaxation in pah molecules as a femto-astrochemistry experiment," Nature Communications 6, 7909 (2015).

${ }^{36}$ M. C. E. Galbraith, C. T. L. Smeenk, G. Reitsma, A. Marciniak, V. Despré, J. Mikosch, N. Zhavoronkov, M. J. J. Vrakking, O. Kornilov, and F. Lépine, "Xuv-induced reactions in benzene on sub-10 fs timescale: nonadiabatic relaxation and proton migration," Phys. Chem. Chem. Phys. 19, 1982219828 (2017).

${ }^{37}$ A. Marciniak, V. Despré, V. Loriot, G. Karras, M. Hervé, L. Quintard, F. Catoire, C. Joblin, E. Constant, A. I. Kuleff, and F. Lépine, "Electron correlation driven non-adiabatic relaxation in molecules excited by an ultrashort extreme ultraviolet pulse," Nature Communications 10, 337 (2019). ${ }^{38}$ A. M. Tokmachev, M. Boggio-Pasqua, D. Mendive-Tapia, M. J. Bearpark, and M. A. Robb, "Fluorescence of the perylene radical cation and an inaccessible d0/d1 conical intersection: An mmvb, rasscf, and td-dft computational study," The Journal of Chemical Physics 132, 044306 (2010).

${ }^{39}$ A. M. Tokmachev, M. Boggio-Pasqua, M. J. Bearpark, and M. A. Robb, "Photostability via sloped conical intersections: A computational study of the pyrene radical cation," The Journal of Physical Chemistry A 112, 10881-10886 (2008).

${ }^{40}$ K. F. Hall, M. Boggio-Pasqua, M. J. Bearpark, and M. A. Robb, "Photostability via sloped conical intersections: A computational study of the excited states of the naphthalene radical cation," The Journal of Physical Chemistry A 110, 13591-13599 (2006).

${ }^{41}$ S. N. Reddy and S. Mahapatra, "Theoretical study on molecules of interstellar interest. i. radical cation of noncompact polycyclic aromatic hydro- 
carbons," The Journal of Physical Chemistry A 117, 8737-8749 (2013).

${ }^{42}$ V. S. Reddy, S. Ghanta, and S. Mahapatra, "First principles quantum dynamical investigation provides evidence for the role of polycyclic aromatic hydrocarbon radical cations in interstellar physics," Physical Review Letters 104, 111102- (2010).

${ }^{43}$ S. Ghanta, V. S. Reddy, and S. Mahapatra, "Theoretical study of the electronically excited radical cations of naphthalene and anthracene as archetypal models for astrophysical observations. part ii. dynamics consequences,' Phys. Chem. Chem. Phys. 13, 14531-14541 (2011)

${ }^{44}$ S. Ghanta, V. S. Reddy, and S. Mahapatra, "Theoretical study of electronically excited radical cations of naphthalene and anthracene as archetypal models for astrophysical observations. part i. static aspects," Phys. Chem. Chem. Phys. 13, 14523-14530 (2011)

${ }^{45}$ E. Tapavicza, I. Tavernelli, and U. Rothlisberger, "Trajectory surface hopping within linear response time-dependent density-functional theory," Phys. Rev. Lett. 98, 023001 (2007).

${ }^{46}$ T. A. Niehaus, S. Suhai, F. Della Sala, P. Lugli, M. Elstner, G. Seifert, and T. Frauenheim, "Tight-binding approach to time-dependent densityfunctional response theory,” Phys. Rev. B 63, 085108 (2001).

${ }^{47}$ J. C. Tully, "Molecular dynamics with electronic transitions," The Journal of Chemical Physics 93, 1061-1071 (1990).

${ }^{48}$ S. Hammes-Schiffer and J. C. Tully, "Proton transfer in solution: Molecular dynamics with quantum transitions," The Journal of Chemical Physics 101 , 4657-4667 (1994).

${ }^{49}$ A. Humeniuk and R. Mitrić, "Dftbaby: A software package for nonadiabatic molecular dynamics simulations based on long-range corrected tight-binding td-dft(b)," Computer Physics Communications 221, 174-202 (2017).

${ }^{50}$ L. Stojanović, S. G. Aziz, R. H. Hilal, F. Plasser, T. A. Niehaus, and M. Barbatti, "Nonadiabatic dynamics of cycloparaphenylenes with td-dftb surface hopping," Journal of Chemical Theory and Computation 13, 5846-5860 (2017).

${ }^{51}$ S. Pal, D. J. Trivedi, A. V. Akimov, B. Aradi, T. Frauenheim, and O. V. Prezhdo, "Nonadiabatic molecular dynamics for thousand atom systems: A tight-binding approach toward pyxaid," Journal of Chemical Theory and Computation 12, 1436-1448 (2016)

${ }^{52}$ E. Posenitskiy, M. Rapacioli, B. Lepetit, D. Lemoine, and F. Spiegelman, "Non-adiabatic molecular dynamics investigation of the size dependence of the electronic relaxation in polyacenes," Phys. Chem. Chem. Phys. 21 , 12139-12149 (2019).

${ }^{53}$ T. Heine, M. Rapacioli, S. Patchkovskii, J. Frenzel, A. M. Köster, P. Calaminici, H. A. Duarte, S. Escalante, R. Flores-Moreno, A. Goursot, J. U. Reveles, D. R. Salahub, and A. Vela, "demonnano," 2009, http://demon-nano.ups-tlse.fr/

${ }^{54} \mathrm{E}$. Holiday and E. Jope, "The ultraviolet spectral absorption of chrysene, its monomethoxy-derivalives and 1: 2 dimethoxychrysene," Spectrochimica Acta 4, 157 - 164 (1950)

${ }^{55} \mathrm{M}$. M. Husain, "Measurement and theoretical characterization of electronic absorption spectrum of neutral chrysene (c18h12) and its positive ion in h3bo3 matrix," Spectrochimica Acta Part A: Molecular and Biomolecular Spectroscopy 68, 156 - 164 (2007).

${ }^{56}$ O. Khorev, C. D. Bösch, M. Probst, and R. Häner, "Observation of the rare chrysene excimer," Chem. Sci. 5, 1506-1512 (2014).

${ }^{57}$ M. Elstner, D. Porezag, G. Jungnickel, J. Elsner, M. Haugk, T. Frauenheim, S. Suhai, and G. Seifert "Self-consistent-charge density-functional tightbinding method for simulations of complex materials properties," Phys. Rev. B 58, 7260-7268 (1998)

${ }^{58}$ D. Porezag, T. Frauenheim, T. Köhler, G. Seifert, and R. Kaschner, "Construction of tight-binding-like potentials on the basis of density-functional theory: Application to carbon,” Phys. Rev. B 51, 12947-12957 (1995).

${ }^{59}$ G. Seifert, D. Porezag, and T. Frauenheim, "Calculations of molecules, clusters, and solids with a simplified lcao-dft-lda scheme," Int. J. Quantum Chem. 58, 185-192 (1996).

${ }^{60}$ B. F. E. Curchod and T. J. Martínez, "Ab initio nonadiabatic quantum molecular dynamics," Chemical Reviews 118, 3305-3336 (2018).

${ }^{61}$ R. Crespo-Otero and M. Barbatti, "Recent advances and perspectives on nonadiabatic mixed quantum-classical dynamics," Chemical Reviews 118 7026-7068 (2018)

${ }^{62}$ G. Granucci and M. Persico, "Critical appraisal of the fewest switches algorithm for surface hopping," The Journal of Chemical Physics 126, 134114
(2007).

${ }^{63}$ J. E. Subotnik, W. Ouyang, and B. R. Landry, "Can we derive tully's surface-hopping algorithm from the semiclassical quantum liouville equation? almost, but only with decoherence," The Journal of Chemical Physics 139, 214107 (2013).

${ }^{64}$ C. Zhu, S. Nangia, A. W. Jasper, and D. G. Truhlar, "Coherent switching with decay of mixing: An improved treatment of electronic coherence for non-born-oppenheimer trajectories," The Journal of Chemical Physics 121 7658-7670 (2004)

${ }^{65}$ R. Mitrić, U. Werner, M. Wohlgemuth, G. Seifert, and V. BonačićKoutecký, "Nonadiabatic dynamics within time-dependent density functional tight binding method," The Journal of Physical Chemistry A $\mathbf{1 1 3}$ 12700-12705 (2009)

${ }^{66}$ M. J. Frisch, G. W. Trucks, H. B. Schlegel, G. E. Scuseria, M. A. Robb J. R. Cheeseman, G. Scalmani, V. Barone, B. Mennucci, G. A. Petersson, H. Nakatsuji, M. Caricato, X. Li, H. P. Hratchian, A. F. Izmaylov, J. Bloino, G. Zheng, J. L. Sonnenberg, M. Hada, M. Ehara, K. Toyota, R. Fukuda, J. Hasegawa, M. Ishida, T. Nakajima, Y. Honda, O. Kitao, H. Nakai, T. Vreven, J. A. Montgomery, Jr., J. E. Peralta, F. Ogliaro, M. Bearpark, J. J. Heyd, E. Brothers, K. N. Kudin, V. N. Staroverov, T. Keith, R. Kobayashi, J. Normand, K. Raghavachari, A. Rendell, J. C. Burant, S. S. Iyengar, J. Tomasi, M. Cossi, N. Rega, J. M. Millam, M. Klene, J. E. Knox, J. B. Cross, V. Bakken, C. Adamo, J. Jaramillo, R. Gomperts, R. E. Stratmann, O. Yazyev, A. J. Austin, R. Cammi, C. Pomelli, J. W. Ochterski, R. L. Martin, K. Morokuma, V. G. Zakrzewski, G. A. Voth, P. Salvador, J. J. Dannenberg, S. Dapprich, A. D. Daniels, O. Farkas, J. B. Foresman, J. V. Ortiz, J. Cioslowski, and D. J. Fox, Gaussian 09 (Revision D.01), Gaussian Inc., Wallingford, CT, 2009.

${ }^{67} \mathrm{~J}$. Frenzel, A. F. Oliveira, N. Jardillier, T. Heine, and G. Seifert, Semi-relativistic, self-consistent charge Slater-Koster tables for densityfunctional based tight-binding (DFTB) for materials science simulations (TU Dresden, 2004-2009).

${ }^{68}$ E. Tapavicza, I. Tavernelli, U. Rothlisberger, C. Filippi, and M. E. Casida, "Mixed time-dependent density-functional theory/classical trajectory surface hopping study of oxirane photochemistry," The Journal of Chemical Physics 129, 124108 (2008).

${ }^{69}$ D. Bonhommeau, A. Viel, and N. Halberstadt, "Dissociative ionization of neon clusters nen, $n=3$ to 14: A realistic multisurface dynamical study," The Journal of Chemical Physics 123, 054316 (2005), https://doi.org/10.1063/1.1953530.

${ }^{70}$ E. Clar and W. Schmidt, "Correlations between photoelectron and ultraviolet absorption spectra of polycyclic hydrocarbons and the number of aromatic sextets," Tetrahedron 31, 2263 - 2271 (1975).

${ }^{71}$ W. Schmidt, "Photoelectron spectra of polynuclear aromatics. v. correlations with ultraviolet absorption spectra in the catacondensed series," The Journal of Chemical Physics 66, 828-845 (1977), https://doi.org/10.1063/1.433961

${ }^{72}$ M. Kasha, "Characterization of electronic transitions in complex molecules," Discuss. Faraday Soc. 9, 14-19 (1950).

${ }^{73} \mathrm{M}$. Chergui, "Empirical rules of molecular photophysics in the light of ultrafast spectroscopy," Pure and Applied Chemistry 87, 12. 525-536 (2015).

${ }^{74}$ B. Stevens, M. F. Thomaz, and J. Jones, "Intersystem crossing in pyrene," The Journal of Chemical Physics 46, 405-406 (1967).

${ }^{75}$ V. Lawetz, G. Orlandi, and W. Siebrand, "Theory of intersystem crossing in aromatic hydrocarbons," The Journal of Chemical Physics 56, 4058-4072 (1972).

${ }^{76}$ K. Schmidt, S. Brovelli, V. Coropceanu, D. Beljonne, J. Cornil, C. Bazzini, T. Caronna, R. Tubino, F. Meinardi, Z. Shuai, and J.-L. Brédas, "Intersystem crossing processes in nonplanar aromatic heterocyclic molecules," The Journal of Physical Chemistry A 111, 10490-10499 (2007).

${ }^{77}$ W. Humphrey, A. Dalke, and K. Schulten, "VMD - Visual Molecular Dynamics," Journal of Molecular Graphics 14, 33-38 (1996). 


\section{Figures from the paper}
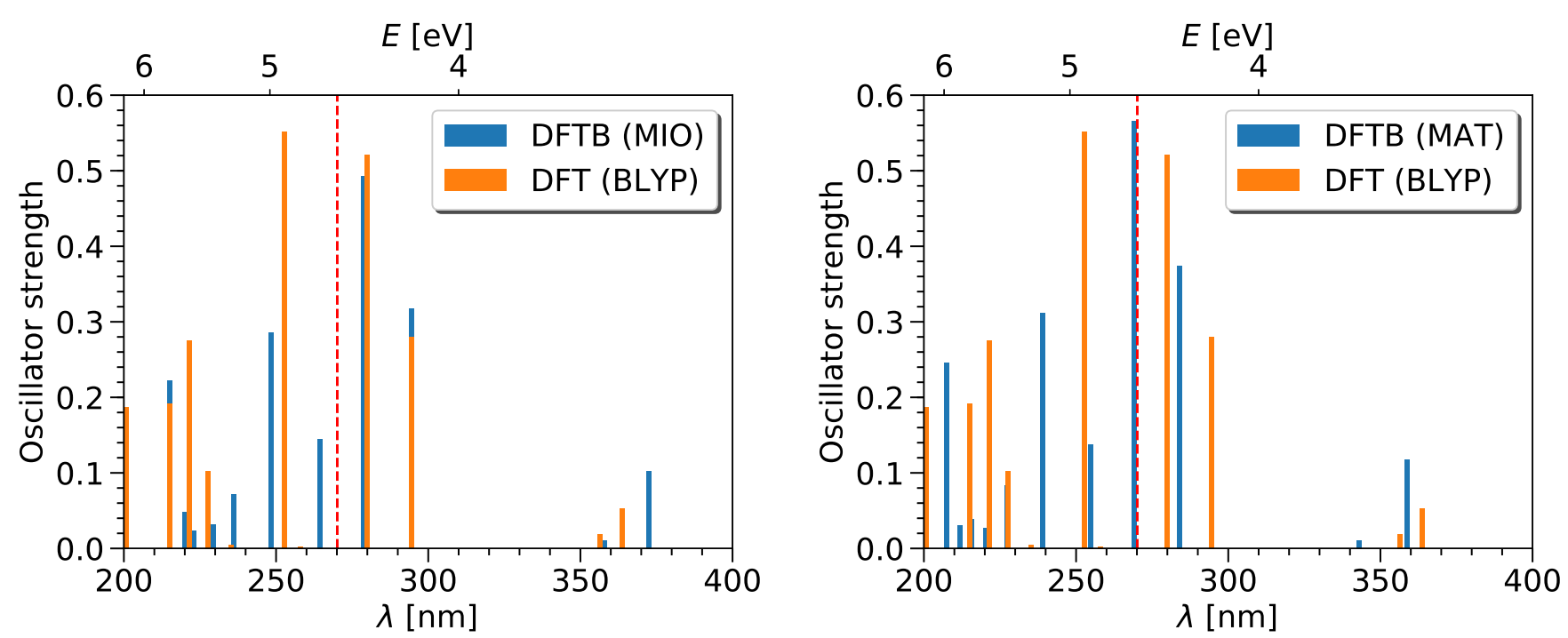

FIG. 1. Absorption spectra (oscillator strengths) of chrysene computed with TD-DFT BLYP functional using 6-31G(d,p) basis set (orange sticks) and TD-DFTB (blue sticks) using mio-1-1 (left panel) and matsci-0-3 parameters set (right panel) at the equilibrium geometry. Red vertical dashed line at $270 \mathrm{~nm}$ indicates the brightest broad band in chrysene (from the experiment in hexane solution ${ }^{54}$ ). Note, that this value is in a good agreement with other experimental measurements: $272 \mathrm{~nm}$ in boric acid matrix ${ }^{55}$ and $269 \mathrm{~nm}^{\text {in tetrahydrofuran solution }}{ }^{56}$.
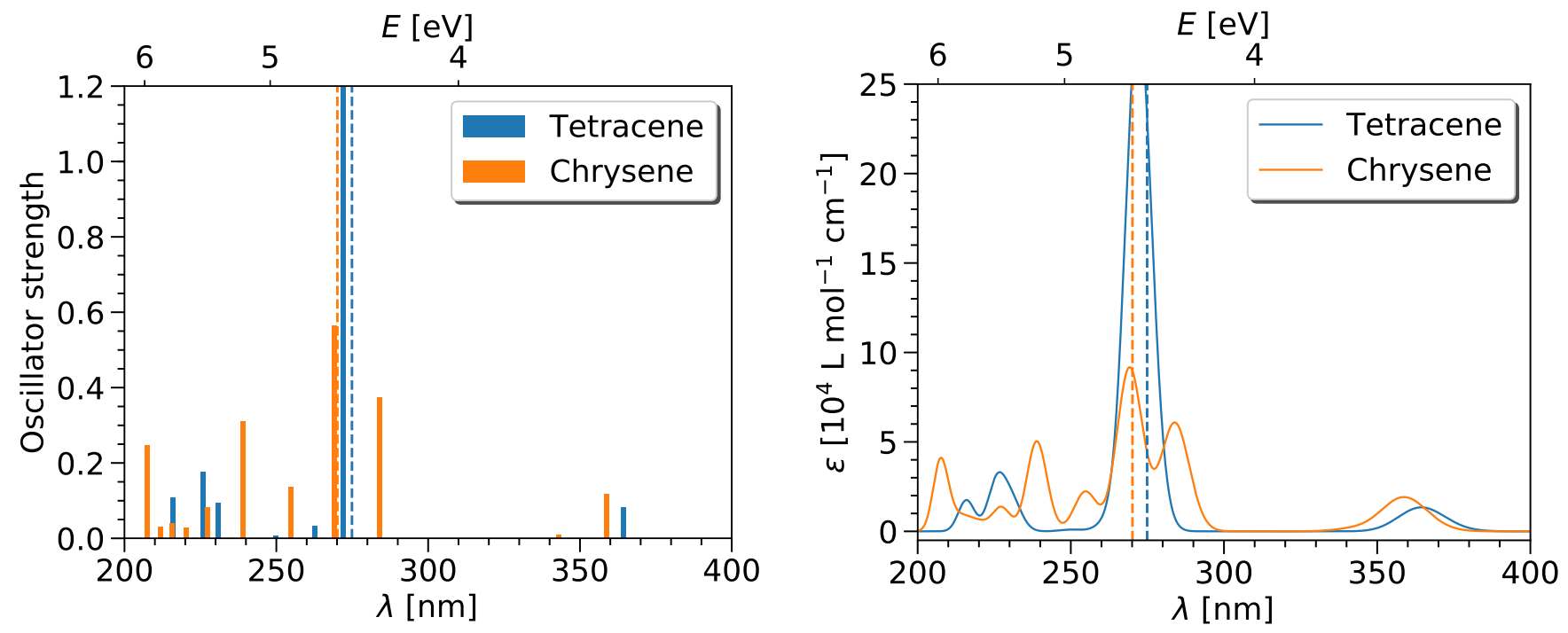

FIG. 2. Oscillator strengths (left panel) and molar absorption coefficients $\varepsilon$ (right panel, convoluted using Gaussian functions with 0.1 eV width) from the UV absorption spectra of chrysene and tetracene computed with TD-DFTB (MAT parameters set) at the equilibrium geometrical configuration. Vertical dashed lines indicate positions of the brightest bands for the corresponding molecule from the experiments. ${ }^{22,54}$ 


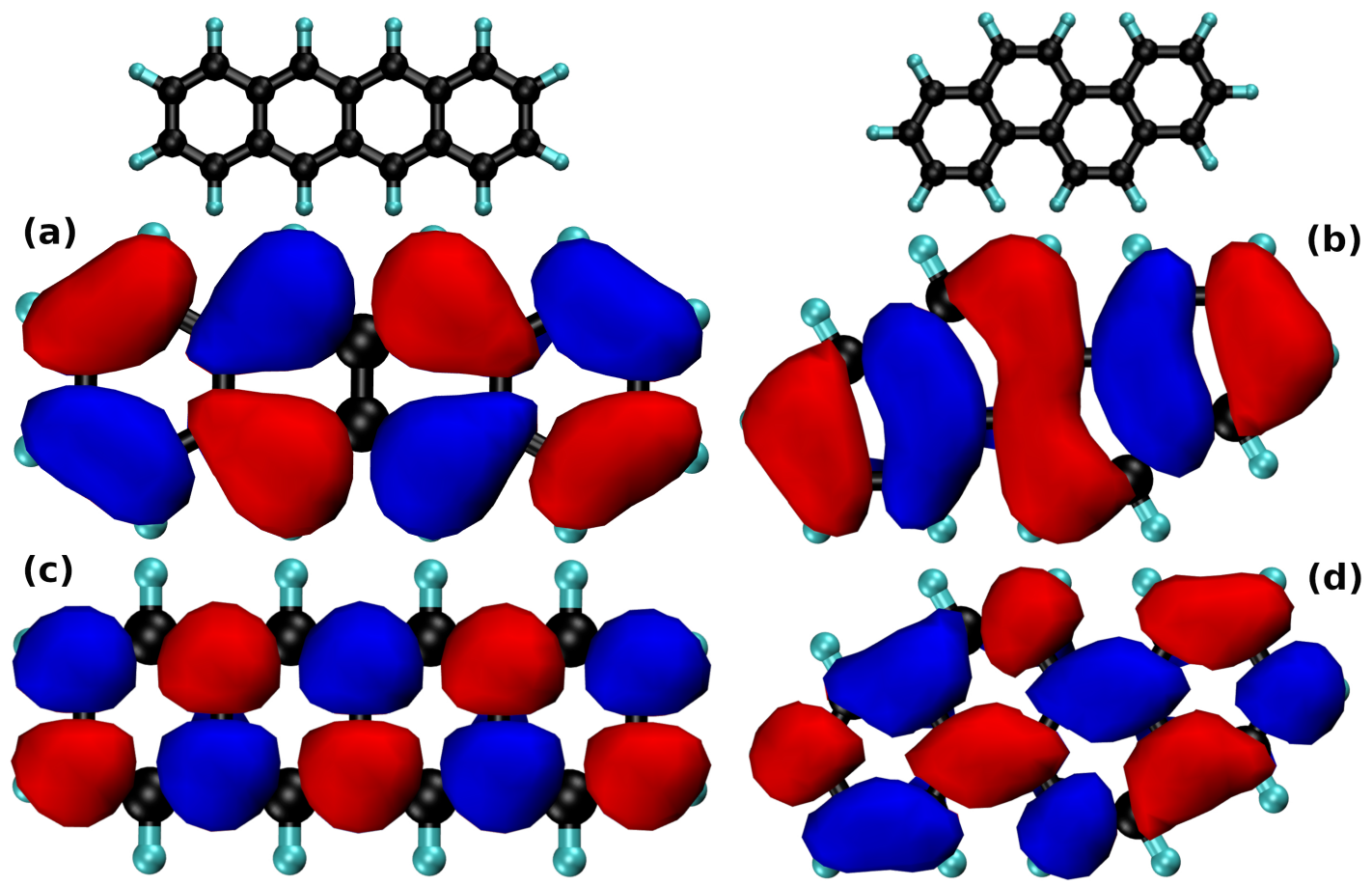

FIG. 3. Isosurfaces of (a) HOMO and (c) LUMO+2 of tetracene (left column); (b) HOMO-1 and (d) LUMO+1 of chrysene (right column). Black and cyan spheres are carbon and hydrogen atoms, respectively. This figure has been produced with VMD. ${ }^{77}$

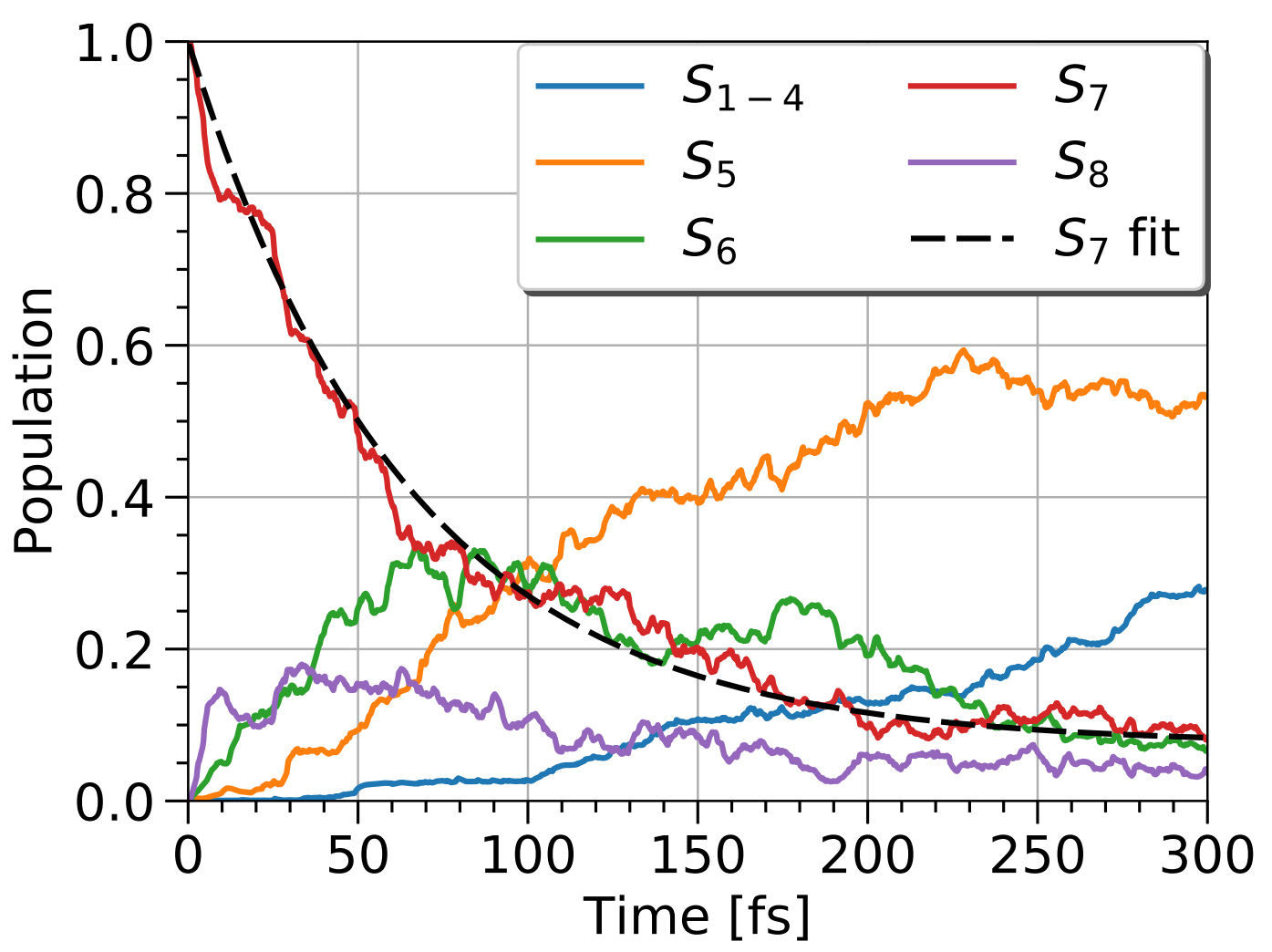

FIG. 4. Population averaged over 100 trajectories in tetracene following excitation to the brightest excited $S_{7}$ state. 


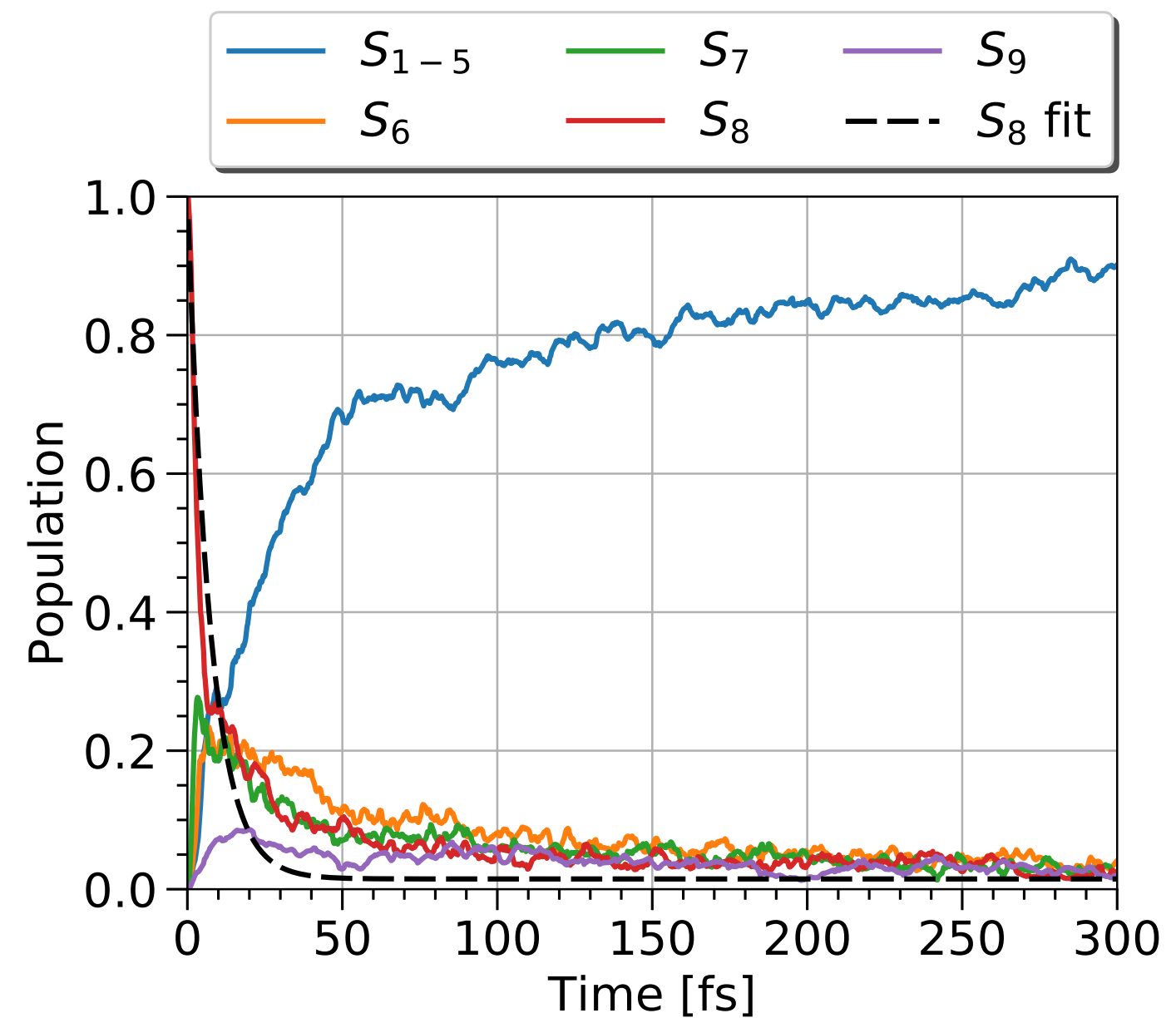

FIG. 5. Population averaged over 100 trajectories in chrysene following excitation to the brightest excited $S_{8}$ state.

Tetracene

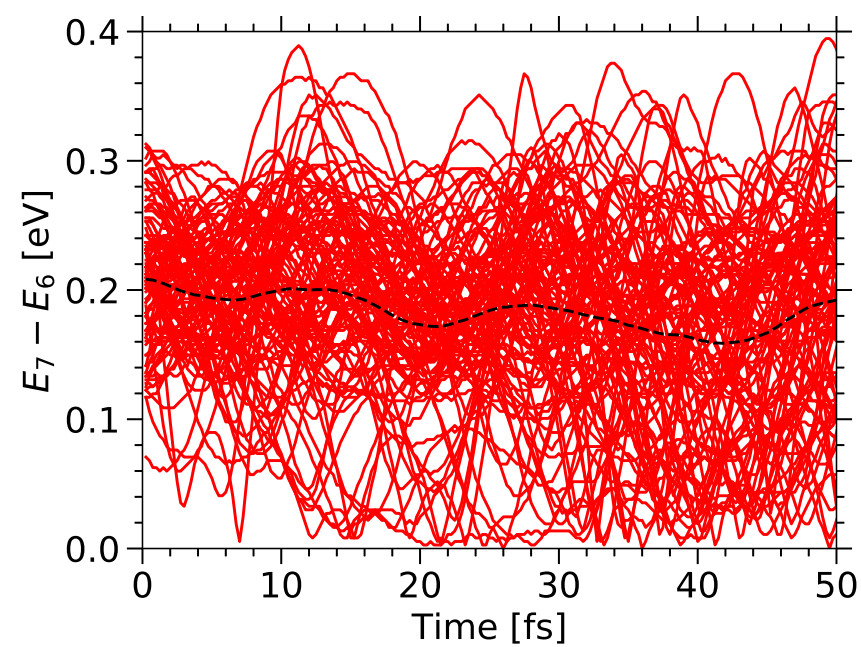

Chrysene

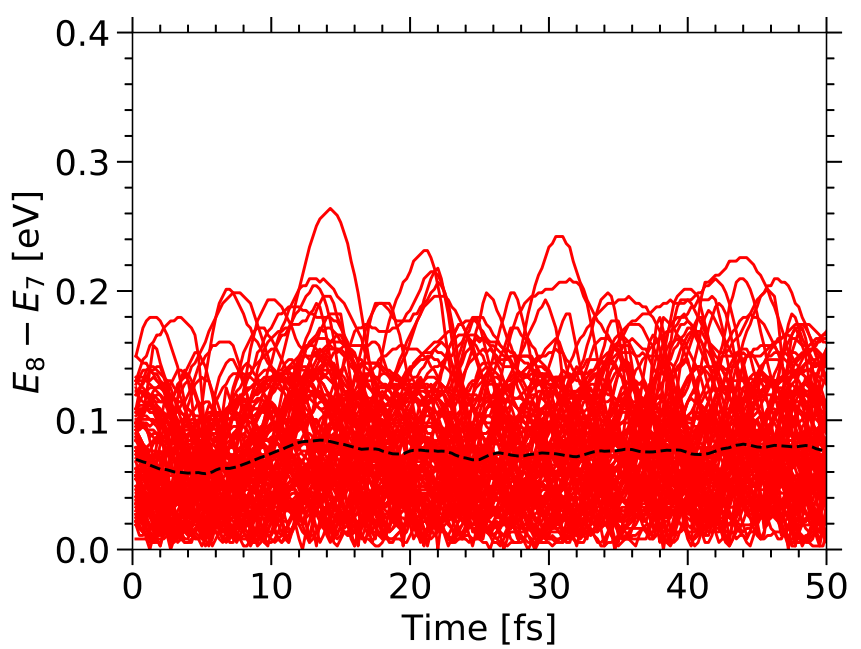

FIG. 6. Energy gaps between the B and B-1 states plotted along 100 trajectories (red lines) and averaged over the entire ensemble (black dashed line) for tetracene and chrysene. 


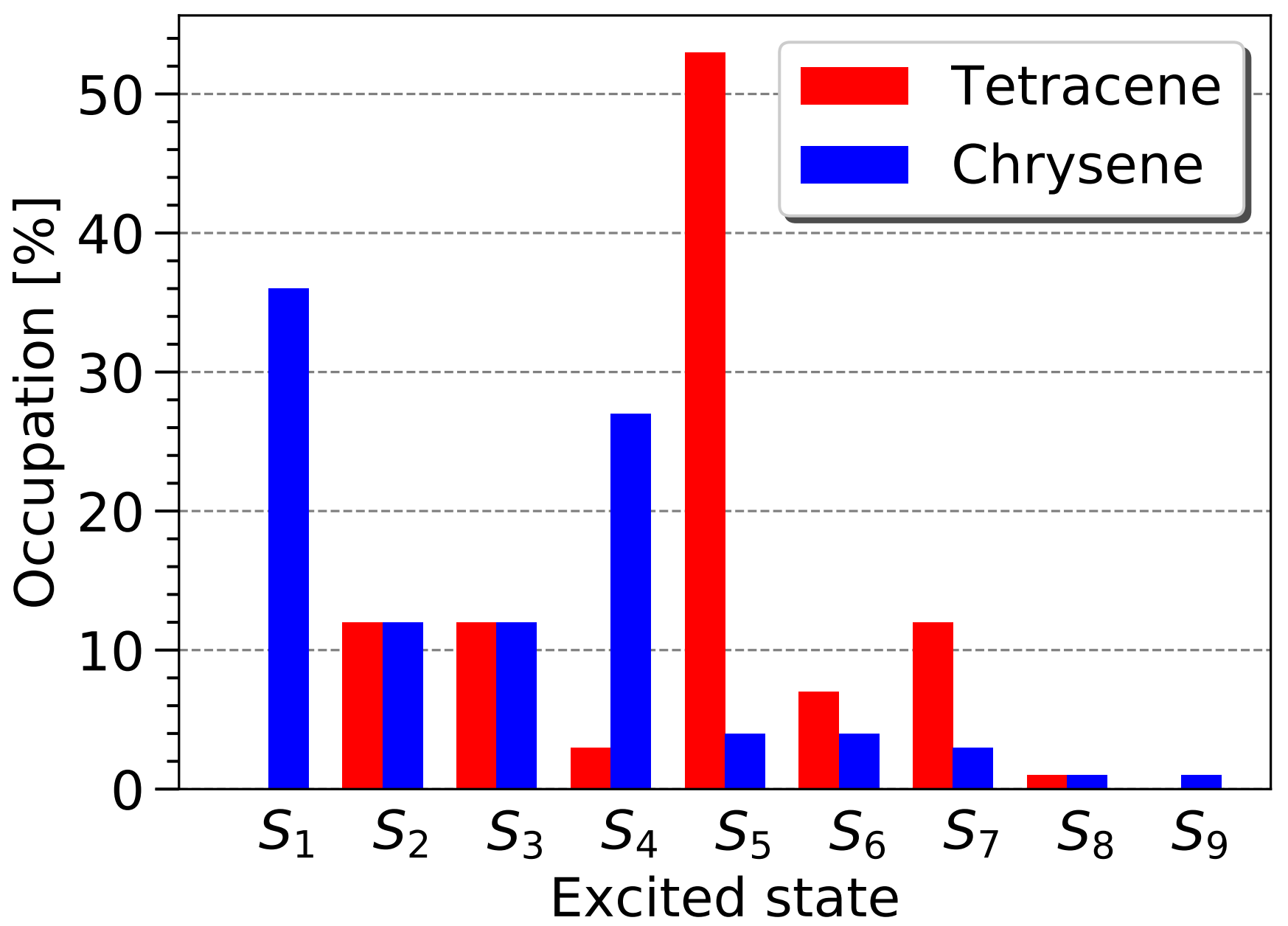

FIG. 7. Occupation of each excited singlet state in tetracene and chrysene after $300 \mathrm{fs}$ of FSSH propagation.
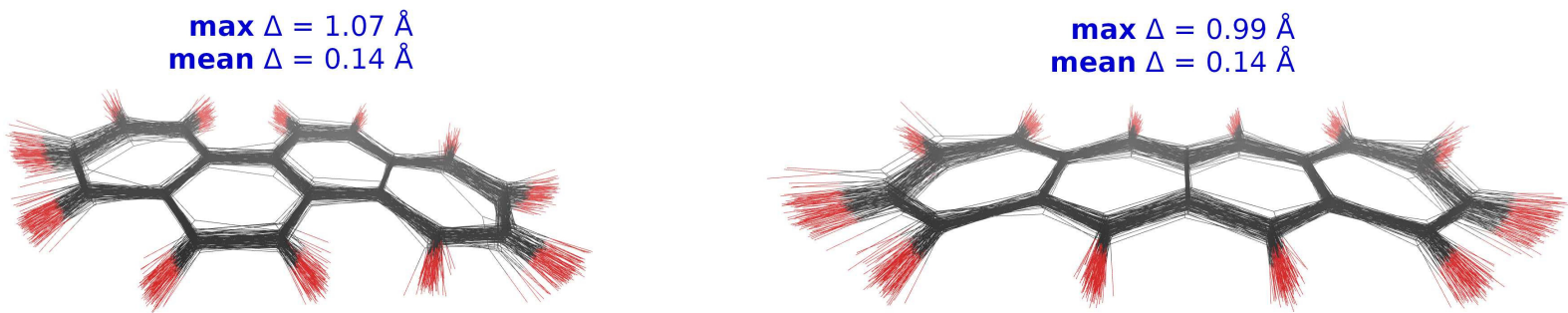

FIG. 8.100 configurations of chrysene (left panel) and tetracene (right panel) at the end of the FSSH simulations. The maximum and mean displacements $(\Delta)$ along the $z$ axis are indicated with respect to the DFTB ground state equilibrium geometry. Black and red colors denote carbon and hydrogen atoms, respectively. 
$E[\mathrm{eV}]$

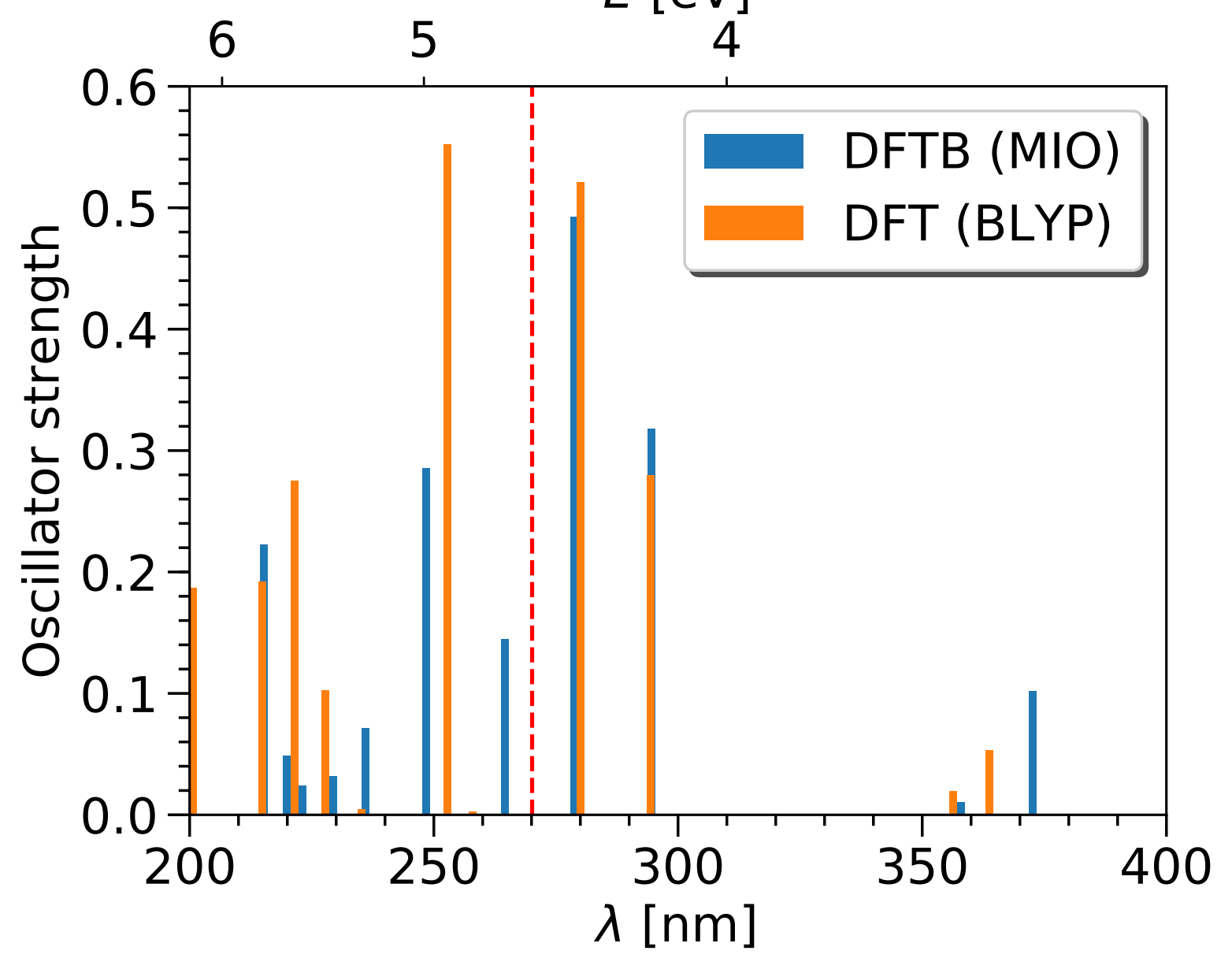


$E[\mathrm{eV}]$

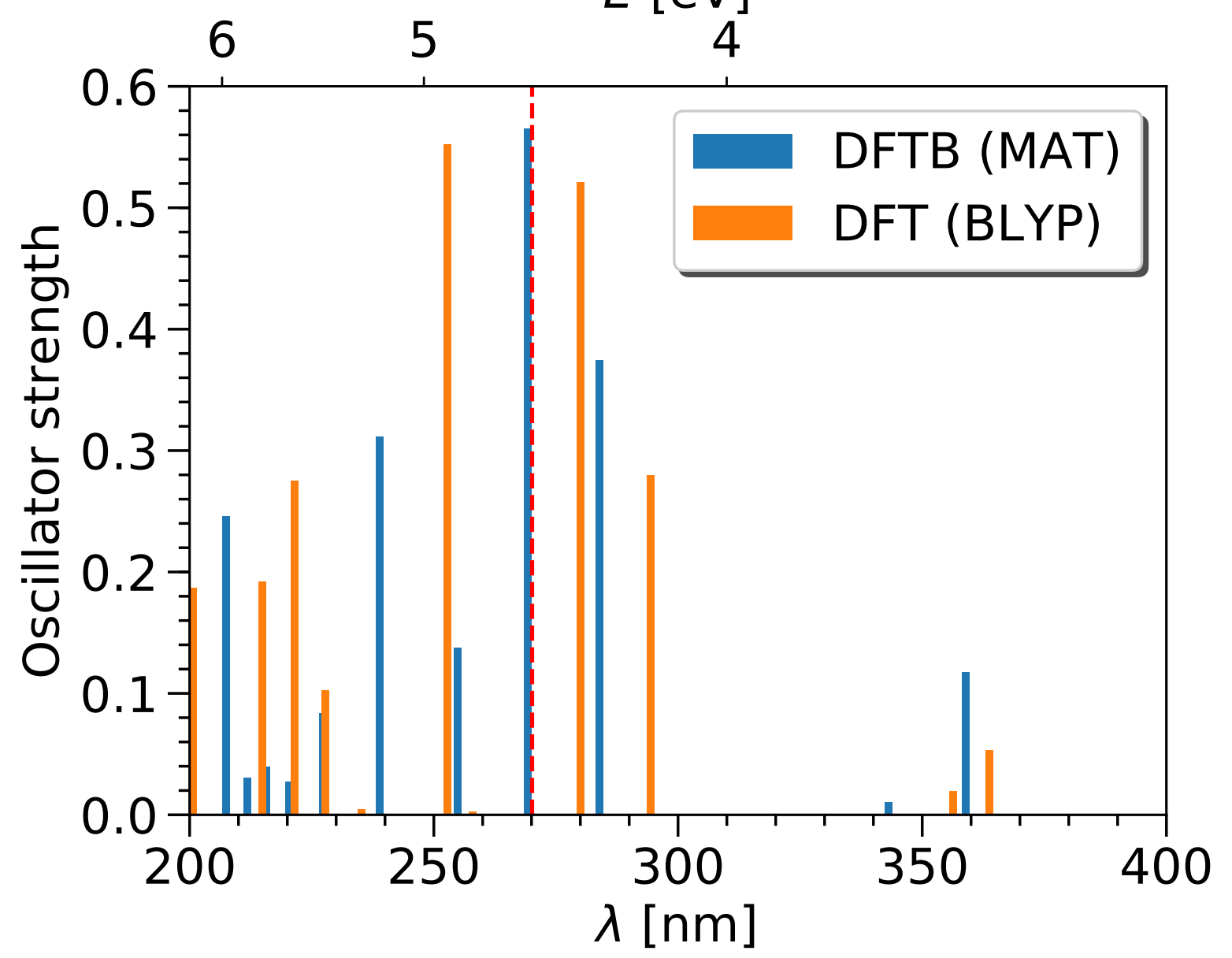




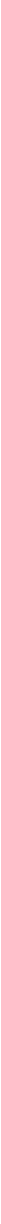




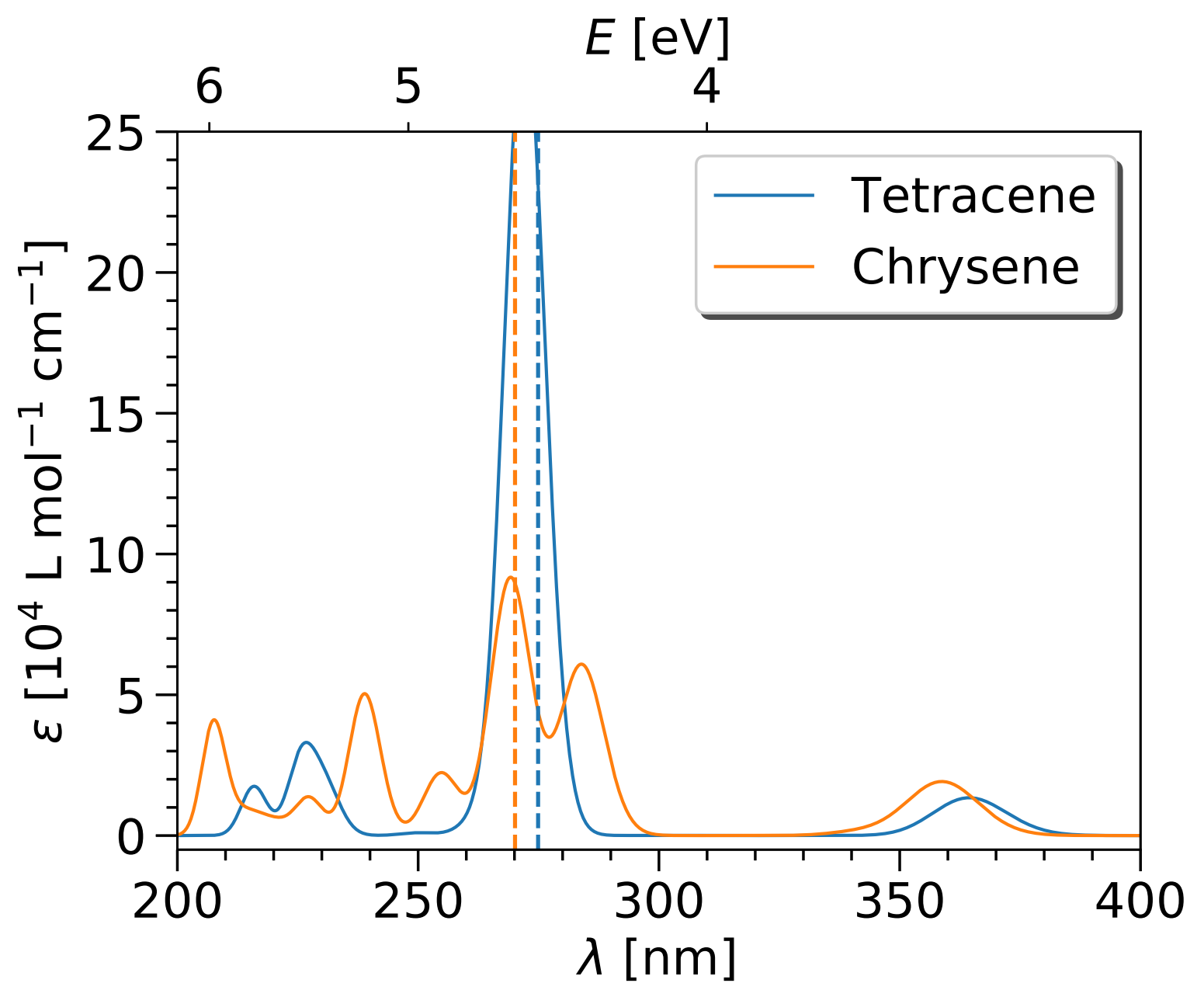




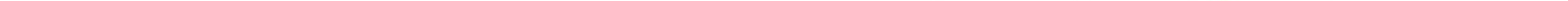




\section{Tetracene}

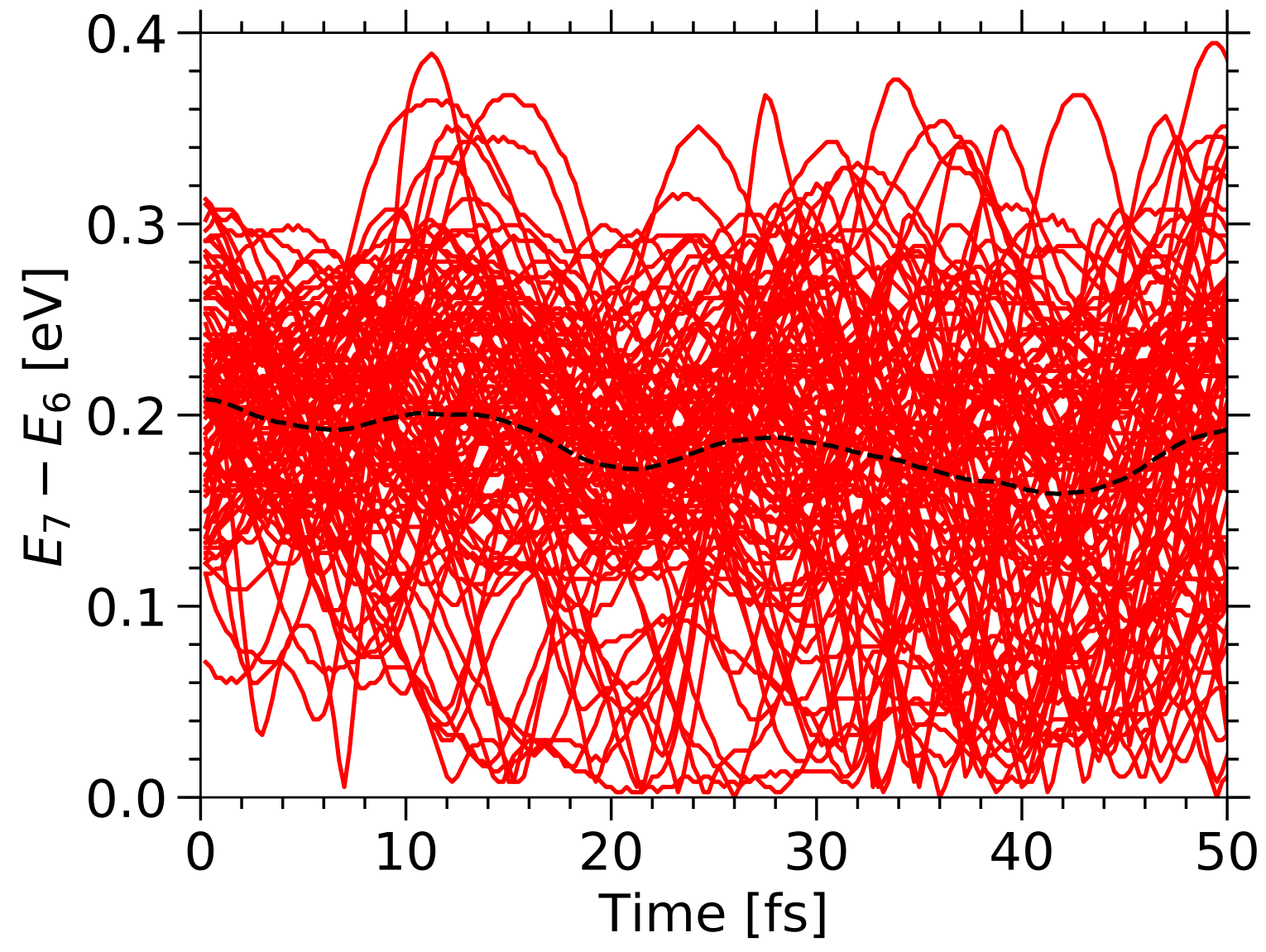




\section{Chrysene}

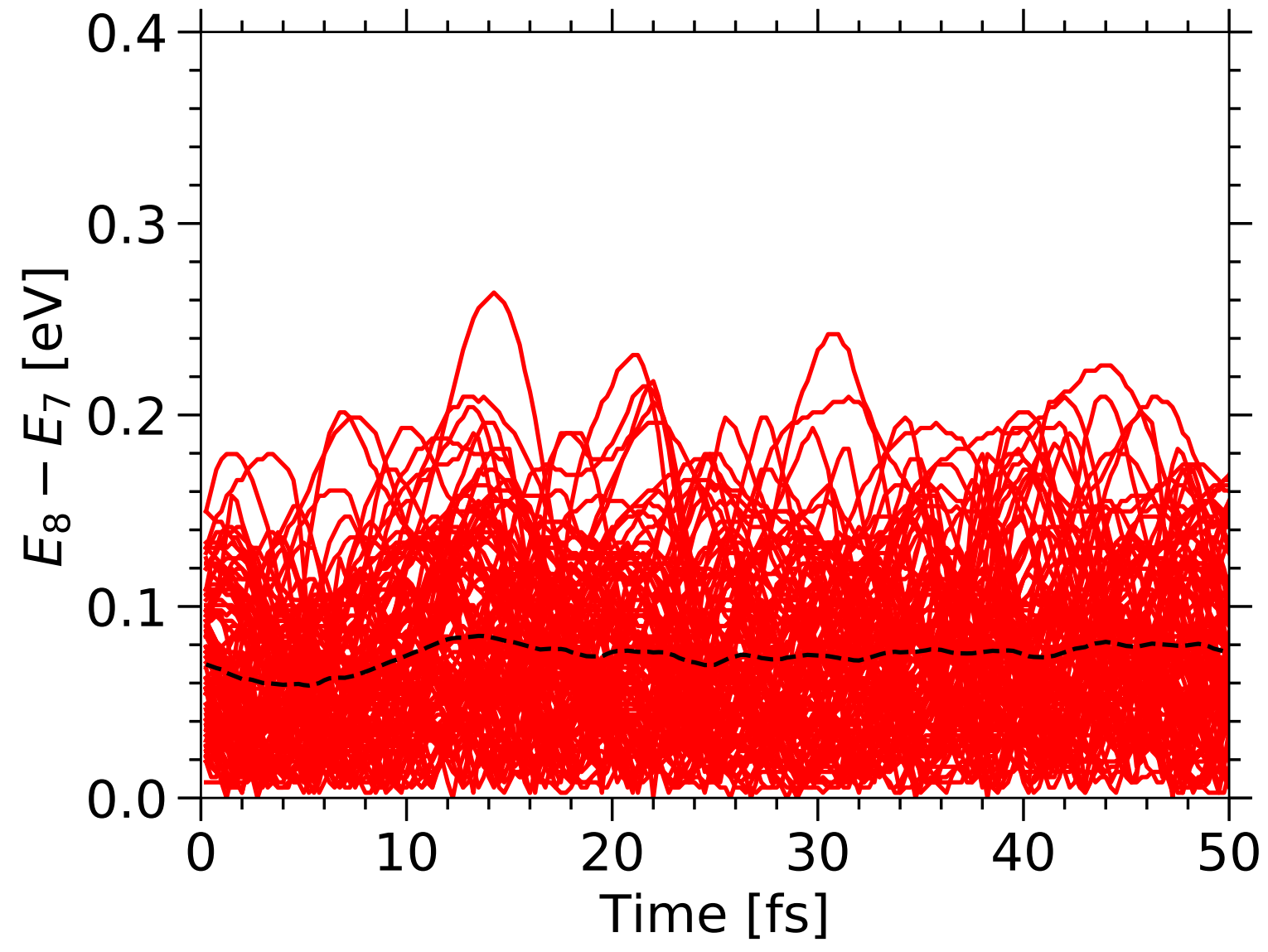




$$
\begin{aligned}
\max \Delta & =1.07 \AA \\
\operatorname{mean} \Delta & =0.14 \AA
\end{aligned}
$$

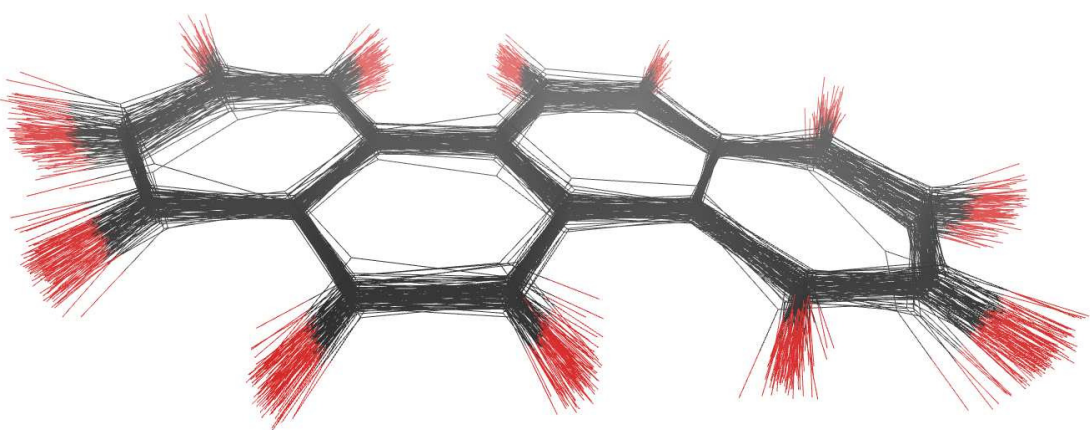




\section{$\max \Delta=0.99 \AA$ mean $\Delta=0.14 \AA$}

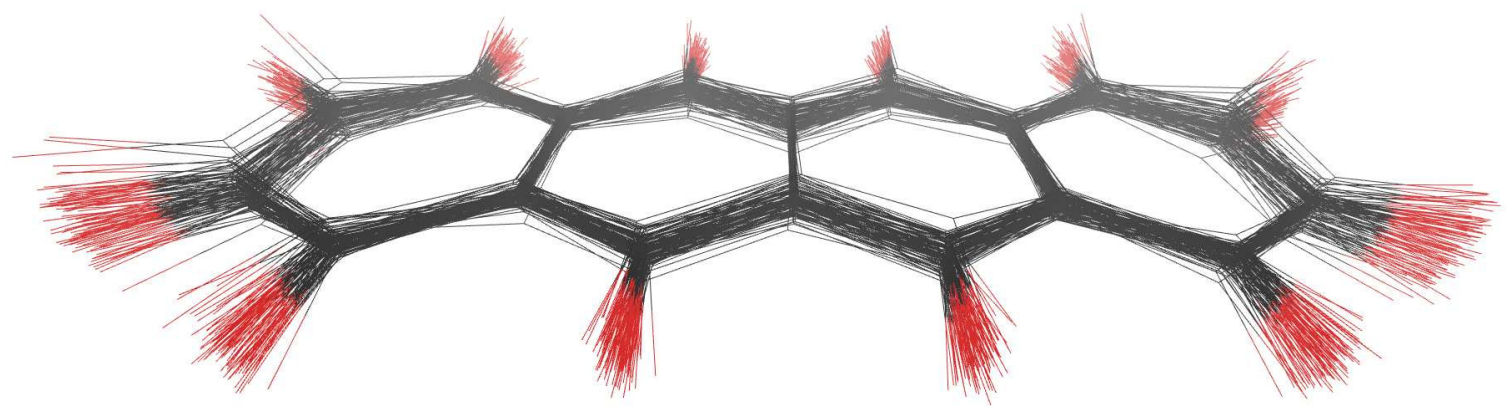

